\title{
IMPORTANCE OF SLIDING WINDOW PROTOCOL
}

\author{
Balwinder Kaur ${ }^{1}$, Mandeep Kaur ${ }^{2}$, Pooja Mudgil ${ }^{3}$, Harjeet Singh ${ }^{4}$ \\ ${ }^{1,2}$ Assistant Professor, Computer Science Department, CT Institute of Technology, Punjab, India, \\ engineer.balwinder@yahoo.in,mandeepquiet@gmail.com \\ ${ }^{3}$ Assistant Professor, Computer Science Department, Bhagwan Parshuram Institute of Technology, Delhi, India, \\ engineer.pooja90@gmail.com \\ ${ }^{4}$ Assistant Professor, Computer Science Department, Lovely Professional University, Punjab, India \\ harjit1985@googlemail.com
}

\begin{abstract}
The data can get lost, reordered or duplicated due to the presence of routers and buffer space over the unreliable channel in the conventional networks. The data link layer deals with frame formation, flow control, error control, and addressing and link management. All such functions will be performed only by data link protocols. The sliding window protocol will detect and correct error if the received data have enough redundant bits or repeat a retransmission of data. The paper shows the working of this duplex protocol of data link network.
\end{abstract}

Keywords: ACK, GOBACK, ARQ, NACK.

\section{INTRODUCTION}

The physical layer deal with transmission signal over different media Data link layer deals with frame formation and flow control, error control over unreliable channels of conventional channel various data limitations cause efficiency decrease. Generally there are two approaches to control such errors:

\section{a) Forward Error Correction (FEC)}

In this the sender adds redundant data to its message known as error-correcting code. This enables the receiver to detect and correct error without repeating additional data from the sender. In this, the back channel is not required and retransmission of data can often be avoided, so it is used where retransmission is either costly or impossible. The FEC systems are designed for simplex channels.

\section{(b) Automatic Repeat Request (ARQ)}

It uses the high rate error-detecting code together with some retransmission protocol. When the error is detected by receiver it generates negative feedback and gives positive feedback for the no error. So this scheme requires a feedback channel.

Similarly the flow control will control, the rate of frame transmission to a value, which can be handled by the receiver. It requires some kind of feedback mechanism. The two basic techniques to achieve flow control accesses data communication links are

a. Stop and wait

b. Sidling window
So In the present paper we will discuss the error correction and flow control function in relation to sliding window. The remaining paper is organized with next section of sliding window overview, significance of senders and receivers windows. A bit sliding window protocol (stop and wait ARQ), a protocol using Go Back $n$,Selective repeat ARQ, Piggybacking and throughout efficiency.

\section{SLIDING WINDOW PROTOCOLS}

This shows a different performance in terms of their efficiency, complexity and buffer requirement. Sliding window protocol assumes full duplex communication. It uses two types of frames, first data and second acknowledgment. One of important features of all the sliding windows protocol is that each outbound frame contains a sequence number, ranging from 0 to $2^{\mathrm{n}}-1$, where the value of $\mathrm{n}$ can be arbitrary. Sliding window refers to imaginary boxes at the transmitter and receiver. This window provides the upper limit on the number of frames that can be transmitted before acknowledgment requirement. Window holds the number of frame to provide above mention limit. The frames which are being transmitted to send are falling in sending window similarly frames to be accepted are store in the receiving window.

This sliding window protocol is widely used in communication. Protocol such as TCP,HDLC and SPX .The poor quality communication channels where the data probably will get duplicated, last or reorder, can provide efficient data transfer with high complexity. This protocol is establishing protocol in ISO-051 Protocol stack. 


\subsection{Significance of Sender's and Receiver's Windows:}

Both the senders and receivers windows are needed not to be of same size. The input sequence obtained from sending host are required to be transmitted via an unreliable network, they must be delivered on the receiving host on the same order in which they appear in the input sequence. So that the correctness condition for sliding window protocol is satisfied.

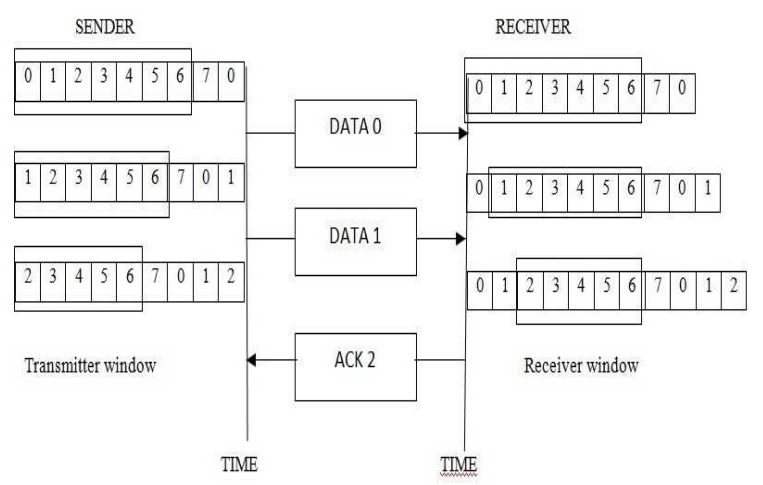

Fig.1 Sliding Windows

The sequence no. within the sender's window gives the number of frame sent but not yet acknowledge. The frames in the sender's window are stored so that they can be possibly retransmitted in the case of damage while travelling to receiver.

The receiver window represents not the number of frame receive but the no. of frame that may still be received before an ACK is sent. Because sliding window of receiver strings from left when frame of data are received and expand to right when ACK is sent. The receiver window contains (n-1) spaces for frame.

\subsection{A One Bit Sliding Window Protocol (Stop and}

\section{Wait ARQ):}

In this case $n=1$ and uses stop and wait technique. Sender waits for ACK after each frame transmission. The operation of this protocol is based on the ARQ(automatic repeat request) principle, which hold the next frame will be transmitted when positive ACK is received and when negative ACK is received, it retransmit the same frame.

Stop and wait ARQ becomes inefficient when the propagation delay is much greater than the time tool retransmit for example let us assume that frame of 800 bits is transmitted over channel with speed $1 \mathrm{mbps}$ and let time for transmission if from end ACK is $30 \mathrm{~ms}$. The number of bits that can be transmitted over this channel is 30,000 bits.
But in stop and wait ARQ only 800 bits can be transmitted as it waits for ACK. The product of bit rate and delay is called delay bandwidth product. It helps in measuring last opportunity in transmitted bits.

\subsection{A Protocol Using Go Back n:}

The sender in this case does not waits for the ACK signal for transmission of next frame. The sender continuously transmits the frame so that the channel should be kept busy rather that wasting time in waiting for it ACK. Because in stop and protocol system does not transmit anything while it is waiting So channel remain idle for considerable time period But in this case the system does depends on only $\mathrm{NACK}$ (negative feedback). It symbolizes error in a particular frame. But as NACK signal will take same time to reach sender, the sender will continue to transmit. On the reception of the NACK signal, the transmitter will retransmit all the frames 3 onwards. The receivers discard all the frames it has received after 3 .

Example: suppose the frame is being transmitted end at frame bit 3 error occurs and NACK is transmitted at the receiver. But this takes some time to reach the transmitter. By the time upto frame 7 has all ways been transmitted.

If the transmitter frame is lost or acknowledgement is lost then only error occurs. In case of damaged or lost frames the receiver transmits NACK to transmitter and the transmitter retransmits all the frames sent since the last frame acknowledged. The disadvantage of go back ARQ protocol is that its efficiency decreases in noisy channel as it does not wait for ACK after every frame transmitted.

\section{PIGGY BACKING}

MOST of the real time systems the data transmission needs to be bi-directional which is popularly called as full duplex system. Now this two way transmission can be achieved in two ways:

a) The method in which two separate channels are involved for both forward and reversed transfer. But in this case acknowledgement are involved which waste the bandwidth of the reverse entirely. This whole system is the basis of the sliding window protocol.

b) But there is a better solution to achieve full duplexing with same capacity channels for both ways (forward and reversed) transmission. In this technique, suppose two A and B users transmits data frame then data frames from $\mathrm{A}$ and $\mathrm{B}$ is intermixed with $\mathrm{ACK}$ and from $\mathrm{B}$ to $\mathrm{A}$. when the receivers receives the data frame it does not transfer ACK signal immediately but wait until the network layer of receiver moves to next data packet then ACK is attached to this outgoing data packet to be transmitted to A. this technique in which the acknowledgement is delayed temporarily is called piggy backing. Though it provides additional complexity but involves better use of channel bandwidth. The only thing we 
have to consider is that data link layer may take too long to transmit.

\subsection{Improvement of the Throughput Efficiency:}

If the delays remain unchanged the throughput efficiency will decrease. So to compensate this it will be necessary to use longer blocks for higher data rates. But it must be kept in mind that the longer blocks will have a greater probability of error. So the optimum block length is must for any particular system so throughput efficiency depends upon the type of system used.

The half duplex system have poor efficiency which can be increased by adapting the continuous mode of transmission instead of block by block transmission. This system will avoid dead time but needs more storage or buffering.

\section{SIMPLE PROCEDURE OF SLIDING WINDOW COMMUNICATION:}

- In the beginning the sending window and the receiving window are empty.

- Sender start sending data frames and receiver waits for frames from network.

- Whenever sender gets new packet from network layer the next higher sequence number according to sending window is given and the upper edge is advanced by one.

- After transmitting one frame and starting the timer, the sender will transmit next data until sending window is filled.

- $\quad$ in mean time sender wait for ACK.

- If active ACK is received then sender fetches next packet and overwrite the previous packet on buffer.

- If damaged ACK or timer goes off then duplicate should be sent.

- When a valid frame arrives, its sequence number is checked to see if it is the next one. If it the next one then it is accepted and passed to network layer and ACK will be generated.

- But if it does not happen so then it will be discarded and is not passed to network layer.

\section{CONCLUSIONS}

In the above paper the brief discussion of the sliding window protocols and associated systems is given. We can develop a general overview easily from the research paper which together has information poured from various sources and persons. We can analyze the advantage and disadvantage of various data link protocols included in the sliding window protocol. We can easily build up concise that computer network will be useless without sliding window protocol because without it, network transmit data over channels that often lose, duplicate or reorder messages.

\section{REFERENCES}

[1]. A.S. Tanenbaum. Computer Networks Prentice-Hall International, Inc., 1996.

[2]. Analysis of sliding window protocol for connected node volume-2, Issue-5, November 2012

[3].N.V. Stenning. A data transfer protocol. Computer Networks, 1(2):99-110, 1976. Eric Madelaine and Didier Vergamini. Specification and Verification of a Sliding Window Protocol in LOTOS FORTE '91, Sydney, Australia, , pages 495-510 November 1991

[4]. Mark A. Smith and Nils Klarlund. Verification of a Sliding Window Protocol Using IOA and MONA FORTE/PSTV 2000, Pisa, Italy, pages 19-34, October 2000. 